\title{
AERO- AND THERMODYNAMIC ANALYSIS TO SHEFEX I
}

\author{
Tarik Barth \\ DLR Institute of Aerodynamics and Flow Technology, Lilienthalplatz 7, D-38108 Braunschweig \\ E-mail:Tarik.Barth@dlr.de
}

\begin{abstract}
The SHarp Edge Flight EXperiment of DLR has been established to demonstrate the feasibility of space vehicles with facetted Thermal Protection System by keeping or improving aerodynamic properties. The TPS consists of simple flat panels with sharp edges and without any constrains in the system compatibility and reliability of space vehicles. This study presents an aero- thermodynamic analysis of the flight of SHEFEX I. To compare the numerical with the experimental data Navier-Stokes calculations have been performed using the flow solver TAU of the DLR. Major effects like influence of sideslip, differences between 2D and 3D simulations, impact of ablation, and so on are analysed to understand the potential source of errors in numerical modelling and their impact on the results. The calculations (pressure and heat flux distributions) for selected altitudes of the reentry offer a well agreement compared with experimental data.
\end{abstract}

Keywords: SHEFEX, re-entry, flight experiment, numerical simulation, heat flux, surface pressure

\section{INTRODUCTION}

Hypersonic systems are complex, difficult to design and expensive to build due to a lack in the physical understanding of the involved flow regimes and a lack of data for design. In order to improve the reliability of accessing space, problems related to vehicle servicing and refurbishing for instance, must be highly simplified and the time required for a design cycle has to be drastically reduced.

The SHEFEX I experiment of DLR was started from northern Norway Andoya Rocket Range on 27. October'05. SHEFEX I flew on top of a twostage solid propellant sounding rocket. The project is a low cost flight experiment, which should demonstrate the feasibility of space vehicles with facetted Thermal Protection System (TPS) by keeping or improving aerodynamic properties. The TPS consists of simple flat panels with sharp edges and without any constrains in the system compatibility and reliability of space vehicles (Fig. 1). The main purpose was to enable the time accurate investigation of the flow effects and their structural answer during the hypersonic flight from $80 \mathrm{~km}$ down to an altitude of $20 \mathrm{~km}$. An additional goal of the experiment was to verify the potential of multidisciplinary design tools. During the flight surface pressures, temperatures and heat fluxes were measured and stored using pressure transducers, thermocouples, heat flux sensors and a pyrometer. Additionally, the output of a Digital Miniature Attitude Reference System (DMARS) platform provides the complete flight mechanic description: Euler angles, accelerations, speed and positions, from lift-off until the deployment of the recovery system.

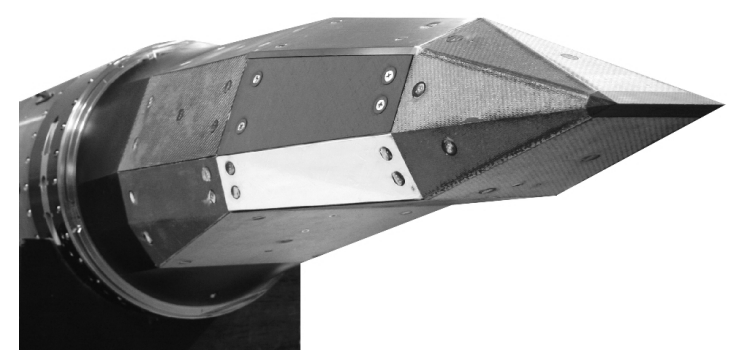

Fig. 1 The SHEFEX I forebody

\section{DESCRIPTION OF THE EXPERIMENT}

The SHEFEX I launcher is a two-stage solid propellant sounding rocket system. The launch vehicle consisted of a Brazilian S30 motor as first stage and an improved Orion motor as second stage. Between the facetted SHEFEX I experiment and the second stage were two cylindrical modules which housed the recovery system, the main electronics, the data acquisition devices, the power supply, and the cold gas system (Fig. 2). 


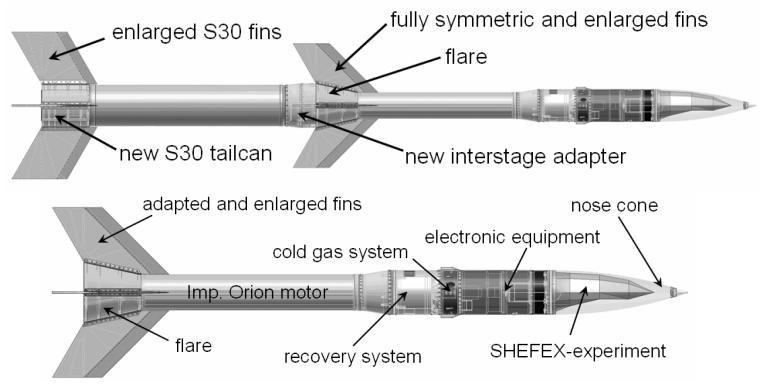

Fig. 2 SHEFEX I launcher re-entry configuration

The vehicle reached an apogee of $211 \mathrm{~km}$. The total flight time was 550 seconds, comprising 45 seconds of experimental time for the atmospheric re-entry between $90 \mathrm{~km}$ and $14 \mathrm{~km}$. The first atmospheric effects on the acceleration sensors could be observed at $80 \mathrm{~km}$. At that level the pitch and yaw angles $(\psi$ and $\Theta)$ started to oscillate and unfortunately the roll rate started to increase, see Fig. 3.

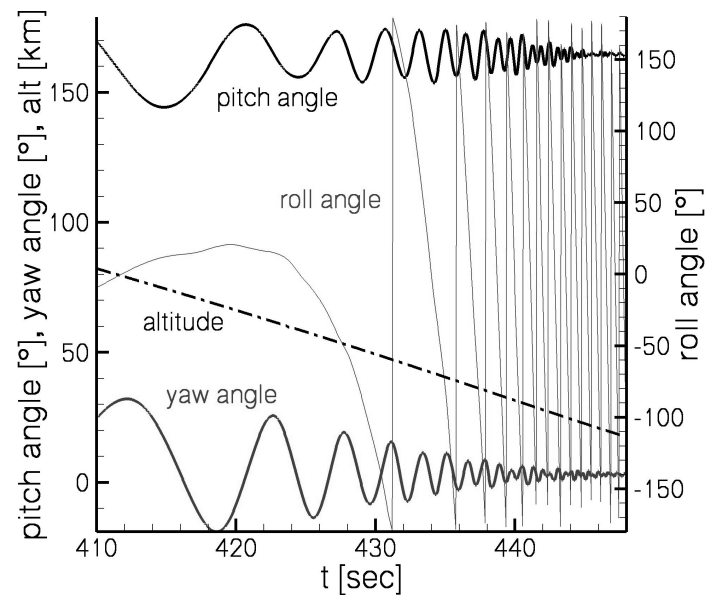

Fig. 3 Re-entry description based on DMARS data

The vehicle finally achieved a stable flight attitude with a decreasing precession around the flight vector. The flight data enable a detailed flight mechanic description of the complete flight. Data of 59 sensors distributed on the surface of the forebody like pressure transducers, thermocouples, and heat flux sensors are available for analysis [Gülhan (2006)].

The flight velocity during the atmospheric descent varied around $1700 \mathrm{~m} / \mathrm{s}$. The Mach number, see Fig. 4, is relatively constant, approx. 5.6 from 100 $\mathrm{km}$ down to $50 \mathrm{~km}$. Then it increases up to a maximum value 6.2 at $26 \mathrm{~km}$.

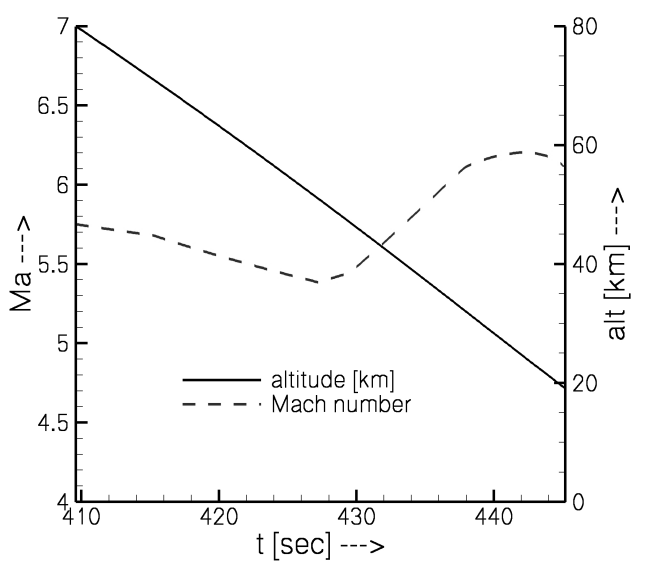

Fig. 4 Flow conditions during re-entry

For the numerical calculations and post-flight analysis the velocity vector is required. This is explicit defined by the transformed angles of attack $\alpha^{\prime}$ and sideslip $\beta^{\prime}$ which already include the effect of the roll angle $\varphi$. A complete and detailed determination of $\alpha^{\prime}$ and $\beta^{\prime}$ is published in [Barth \& Eggers (2007)]. Here below the main results are summarized.

The influence of the roll angle $\varphi$ on the nominal values of the angles of attack $\alpha$ and sideslip $\beta$ is realized transforming their values according to equations (1) and (2).

$$
\begin{aligned}
& \alpha^{\prime}=\cos (\varphi) \cdot \alpha+\sin (\varphi) \cdot \beta \\
& \beta^{\prime}=\cos (\varphi) \cdot \beta-\sin (\varphi) \cdot \alpha
\end{aligned}
$$

Additionally, in order to correct the angles of attack for higher altitudes the oblique shock relations given by equations (3) and (4) is applied.

$$
\begin{gathered}
\tan \mathrm{\nu}=2 \cdot \cot \sigma \cdot\left(\frac{M_{1}{ }^{2} \cdot \sin ^{2} \sigma-1}{M_{1}{ }^{2} \cdot(\kappa+\cos 2 \sigma)+2}\right) \\
\frac{p_{2}}{p_{1}}=\frac{2 \cdot \kappa \cdot M_{1 n}{ }^{2}-(\kappa-1)}{\kappa+1} \\
\text { with } M_{1 n}{ }^{2}=M_{1} \cdot \sin \sigma
\end{gathered}
$$

Here, $p$ is the pressure, $M$ is the Mach number, $\kappa$ is the adiabatic exponent, $v$ is the deflection angle, $\sigma$ is the shock angle, index " " is the condition before the shock, and index "2" is the condition after the shock.

In conclusion, Fig. 5 shows the transformed and corrected angles of attack and sideslip which are used in the present study. 


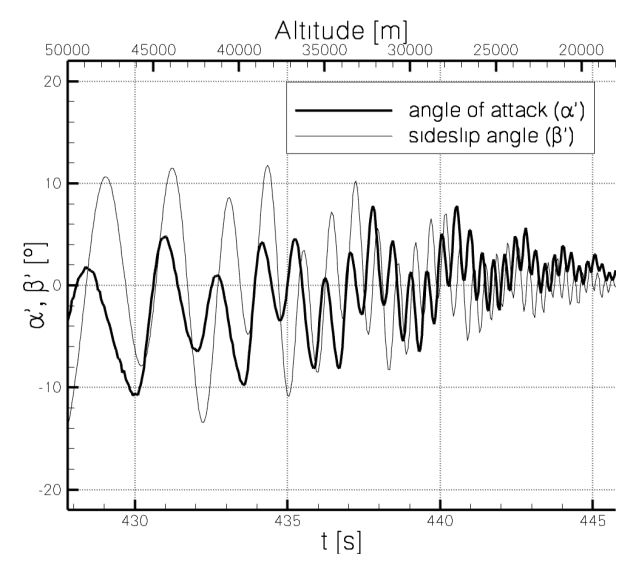

Fig. 5 Transformed and corrected angles of attack and sideslip [Barth \& Eggers (2007)]

\section{NUMERICAL RESULTS}

\subsection{General comments}

Flow solver

For the numerical simulations Navier-Stokes calculations are performed using the flow solver TAU of the DLR. TAU is a three-dimensional parallel hybrid multigrid code. It is a finite volume scheme for solving the Euler and Reynolds-averaged Navier-Stokes (RANS) equations using tetrahedrons and prisms. Prismatic elements were used for boundary layer regions while the tetrahedral elements were used primarily in inviscid flow layer regions. The AUSMDV second order upwind scheme with MUCL reconstruction is used for the inviscid fluxes. For acceleration, multigrid and explicit residual smoothing are available. For time discretization, including local time stepping, a three dimensional Runge-Kutta is implemented. The TAU code includes different turbulence models. In the present case, the two-equation $k-$ $w$ model was used, because other studies ([Schramm \& Reimann (2007)] and [Dankert \& Otto (2006]) pointed out that the results until Mach 8 will be calculated satisfy using this turbulence model. The 3D calculations have been solved on a cluster using 8 processors. A converged solution is achieved after approximately 10,000 iteration steps and 12 hours calculation time respectively.

\section{Numerical discretization}

To generate the grids from the CAD data, the commercial CENTAUR mesh generator was used. The Navier-Stokes mesh had 30 prismatic layers at the wall. The height of the first cell is selected in such way, that $\mathrm{y}^{+}<1$ for all simulations. The mesh consists of approximately 2,000,000 points (after adaptations). Grid adaptation by cell division offers the possibility to insert additional points only in regions where clustering is necessary. For instance, along the shock front high pressure, density, and velocity gradients are existent and a clustering is recommended. While the tetrahedra can be divided along all edges, the prismatic elements are presently refined only on their triangular faces. Two adaptation steps have been used for the present study. Fig. 6 shows the grid after the second adaptation and the prismatic layer.

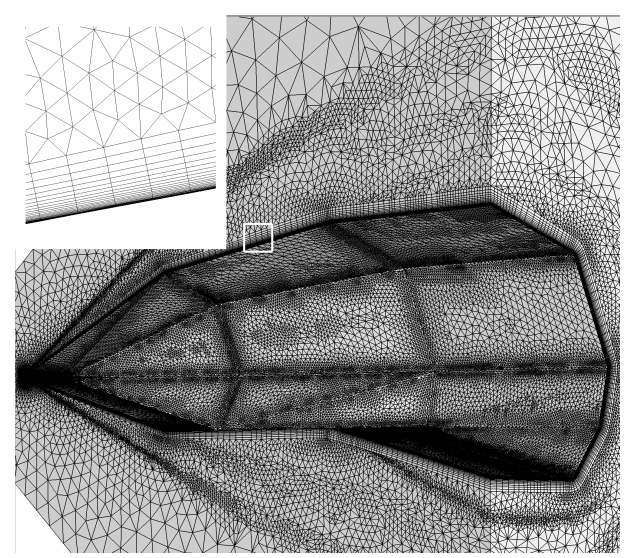

Fig. 6 Numerical grid after a second adaptation. Left above: Zoom of the prismatic elements

\section{Numerical strategy}

Since no transition criteria is implemented in TAU, between 26 and $36 \mathrm{~km}$ an engineering approach is used, for the simulations the flow is initialised as laminar as well as turbulent. Transition is deduced by comparing the computed heat fluxes with the measurement data. In subsection 3.2 the differences between laminar and turbulent boundary layer and their results will be discussed in detail.

The comparisons of experimental and numerical pressure and heat flux distributions are done along two lines on the lower surface (line 1 for pressure and line 2 for heat flux), which meet the exact position of the sensors in the vehicle (Fig. 7).

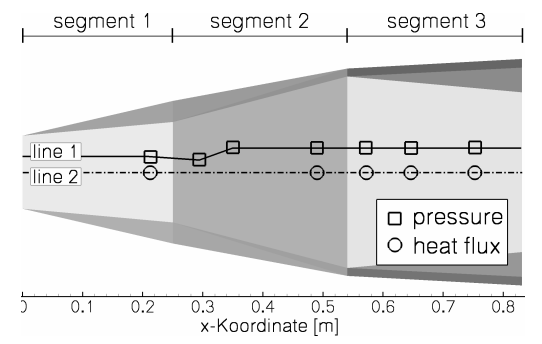

Fig. 7 Position of the pressure and heat flux sensors 


\subsection{Sensitivity analysis}

In the following the accuracy of the data recording during the flight and potentials source of error for the numerical simulations like influence of sideslip, differences between 2D and 3D simulations, impact of ablation, and so on, are investigated and discussed.

\section{Data recording}

At first, the accuracy of the data recording during the experiment is analysed. The experimental (pressure, heat flux, etc.) and flight (position, acceleration, etc.) data were stored every 50 milliseconds. However, these data are measured by different systems. In order to examine the synchronous of the data recording the pressure distribution of the experiment are compared with the numerical results considering the current flight data. The numerical calculations are obtained solving 3D Navier-Stokes equations. The boundary layer is assumed turbulent and the wall behaviour is defined as radiation adiabatic $(\varepsilon=$ 0.94). Table 1 shows the selected time range.

Table 1 Selected times of re-entry and their parameter

\begin{tabular}{|c|c|c|c|c|}
\hline $\mathbf{t}[\mathbf{s}]$ & Alt $[\mathrm{km}]$ & $\mathbf{M}[-]$ & $\boldsymbol{\alpha}^{\prime}\left[{ }^{\circ}\right]$ & $\beta^{\prime}\left[{ }^{\circ}\right]$ \\
\hline 442.85 & 23.21 & & 5.0 & 0.7 \\
\hline 442.90 & 23.12 & \multirow{2}{*}{6.2} & 3.3 & 2.2 \\
\hline 442.95 & 23.03 & & 1.1 & 1.7 \\
\hline
\end{tabular}

Within this time range the pressure changes clearly resulting on the distinct angle of attack gradients. The experimental and numerical results for the selected times are shown in Fig. 8.

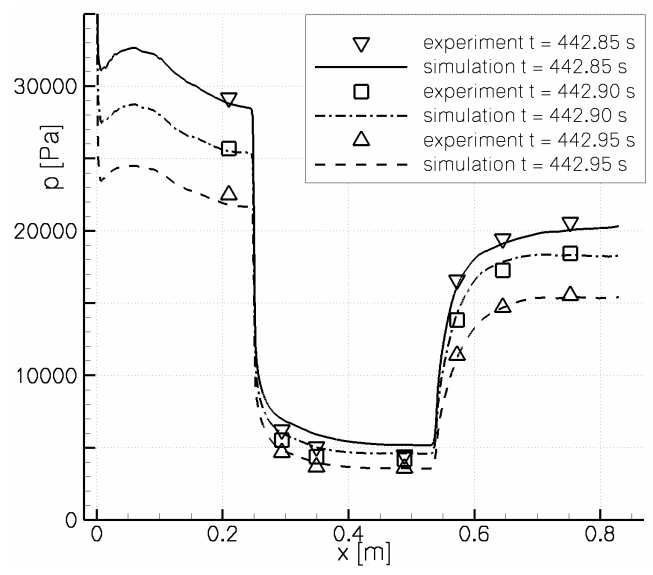

Fig. 8 Numerical and experimental results of the pressure distribution on the lower surface of SHEFEX I

The experimental and numerical data are very close to each other and the gradients are equal. As a result of this comparison it can be summarized that the data recording of the experimental and flight data was carried out synchronously.

\section{Influence of sideslip angle}

In order to save calculation time it is proposed to compute only half of the SHEFEX I forebody. This is only possible when:

- The influence of the sideslip angle is marginal.

- A symmetric flow field may be assumed.

Therefore the influence of the sideslip angle is investigated. The results point out, that a sideslip angle of less than $3^{\circ}$ has only minor influence and a half configuration may be used in these cases. In case of larger values of sideslip the complete body has to be considered because the characteristic of the pressure distribution change (Fig. 9).
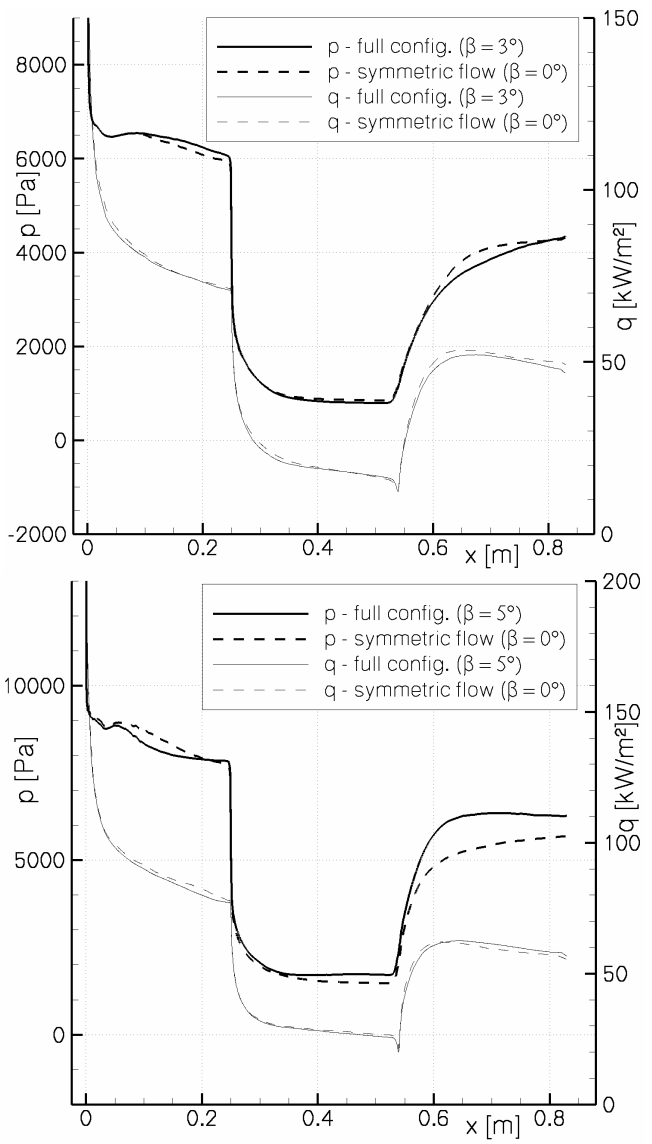

Fig. 9 Numerical and experimental pressure and heat flux distributions - influence of the sideslip angle (top: $\beta=3^{\circ}$, bottom: $\beta=5^{\circ}$ ); Alt $=35 \mathrm{~km}, M=6, \alpha=0^{\circ}$

In opposite to that, the heat flux distribution is largely independent of the sideslip angle, because the sensors are positioned along the symmetry of SHEFEX I (see Fig. 7). 


\section{Comparison between 2D and 3D}

Due to the fact that the sensors are located within and in the near of the symmetry plane respectively the possibility to perform the study only with a $2 \mathrm{D}$ configuration is analysed. If 2D simulations are possible, much calculation-time may be saved. For this purpose the following both points have to conform:

- The results of the 2D and 3D simulations have to be equal along the symmetry line.

- It has to be possible to neglect the offset of the symmetry plane of the pressure sensors (see Fig. 7 for details).

Fig. 10 shows a comparison of results obtained using $2 \mathrm{D}$ and $3 \mathrm{D}$ configurations.
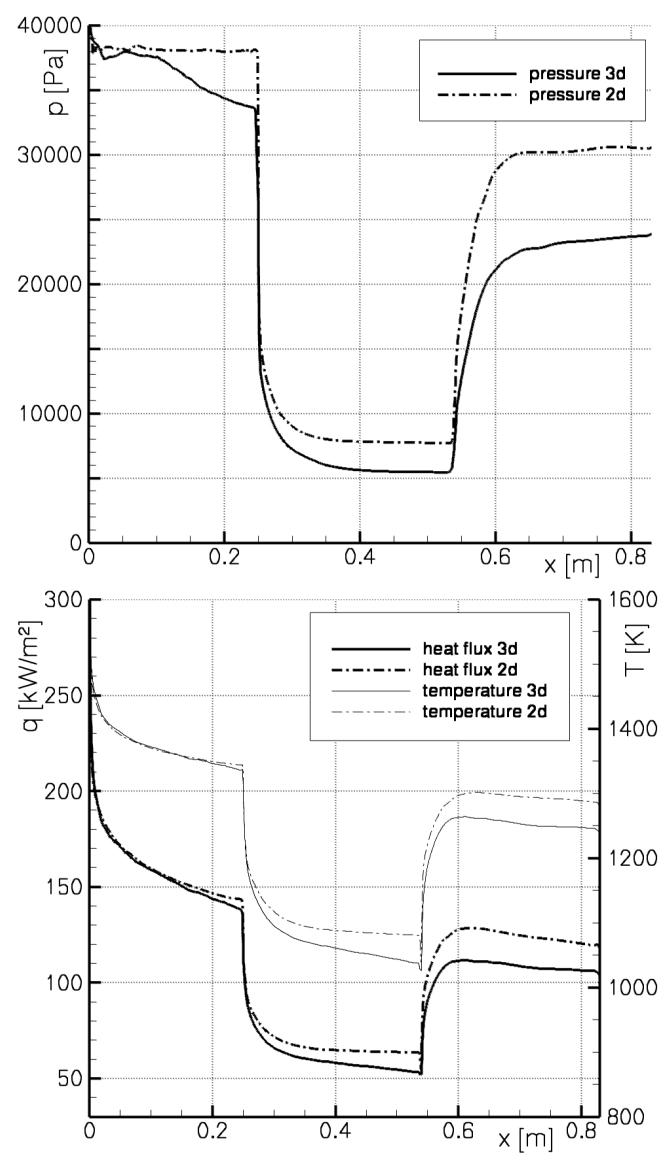

Fig. 10 Comparison of $2 \mathrm{D}$ and $3 \mathrm{D}$ calculations: pressure (top), heat flux and temperature (below); Alt = $20 \mathrm{~km}, \mathrm{M}=6.2, \alpha=0^{\circ}$

The results of the comparison may be discussed as followed:

- The surface pressure distribution can only be calculated correctly using a 3D configuration, mainly due to the offset of the sensors.

- The heat flux and temperature distributions are only nearly identical for 2D and 3D simulations along the first segment of SHEFEX I. After that a 3D configuration has to be taken into account in order to obtain the correct results.

\section{Impact of Ablation}

The next problem is the geometry of the leading edge of SHEFEX I. An absolute sharp edge is with respect to manufacture tolerances not feasible. Therefore, an initial constrains for the leading edge of $\mathrm{R}=0.02 \mathrm{~mm}$ is assumed. Also, during the re-entry flight a change of the geometry as a result of high temperature (ablation) would be possible. Two additional radii $(0.2$ and $2 \mathrm{~mm})$ are investigated on the basis of pressure and heat flux distributions on the lower surface in order to detect the influence of the rounding due to ablation.
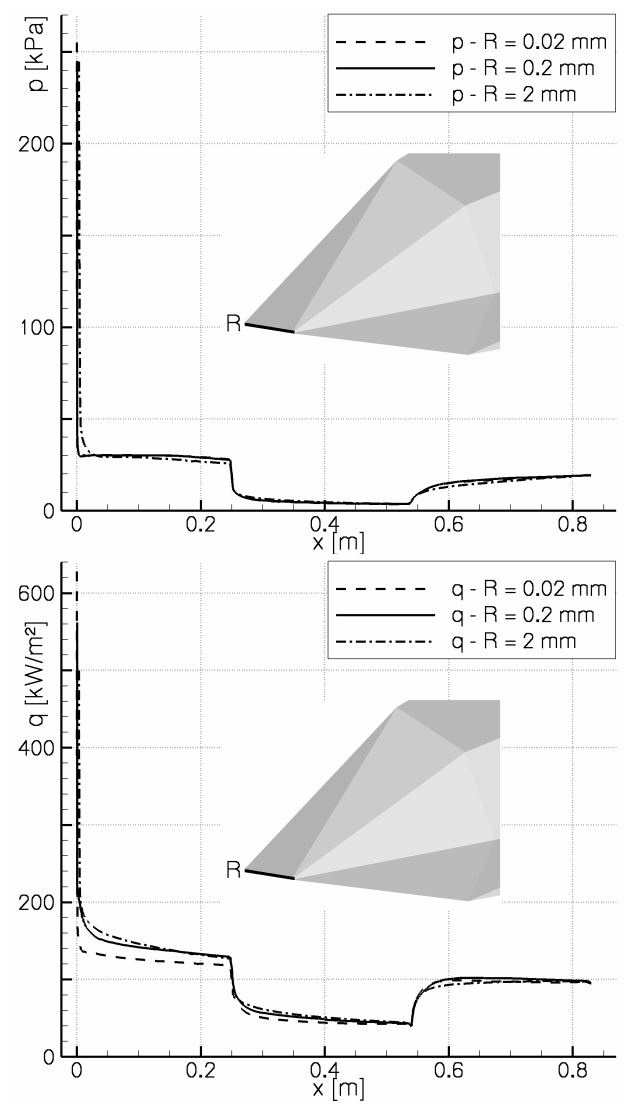

Fig. 11 Influence of the leading edge ablation on pressure (top) and heat flux (below) distributions (Alt = $21 \mathrm{~km}, \mathrm{M}=6.2, \alpha=0^{\circ}$ ) 
Fig. 11 shows the results of the investigated cases. According to these calculations it may be noted that rounding of the leading edge as a consequence of ablation has nearly no impact on the surface pressure. The differences at the stagnation point are smaller than $4 \%$. However, this does not apply to the heat flux distribution. The heat flux differs at the stagnation point about $20 \%$. Additionally, along the first third of SHEFEX I the heat flux distribution depend on the nose radii. In summary it can be ascertained that with increasing ablation (radius) the heat flux on the leading edge decreases and downstream increases.

\section{Effect of atmosphere model}

In the following the effects of the atmosphere model to reduce the flight data are investigated. Former projects, like ARD [Johnston (2002)], pointed out that the applied standard atmospheres had a significant influence on the aerodynamic behaviour and on the aerodynamic coefficients. Therefore, also for the SHEFEX I analysis, it is worth to obtain an overview about the possible uncertainties resulting from different standard atmospheres.

For the comparison, the atmosphere models US Standard Atmosphere 1976, GRAM-99 ${ }^{l}$, and CIRA $86^{2}$ are selected. In account of the fact that SHEFEX I was launched on October $27^{\text {th }}$, thus at the end of October, for the CIRA model the impact of the calendar month is also investigated. In Table $\mathbf{2}$ all values of the models are summarized.

Table 2 Atmosphere models and their values for atmosphere pressure and temperature at $\mathbf{2 0} \mathbf{~ k m}$

\begin{tabular}{|l|c|c|}
\hline \multicolumn{1}{|c|}{ Atmosphere model } & $\mathbf{p}[\mathbf{P a}]$ & $\mathbf{T}[\mathbf{K}]$ \\
\hline US Standard Atmosphere 1976 & 5475 & 216.7 \\
\hline GRAM-99 & 5580 & 206.5 \\
\hline CIRA $86,70^{\circ}$ N, Oct. & 5194 & 217.1 \\
\hline CIRA $86,70^{\circ}$ N, Nov. & 4940 & 212.2 \\
\hline
\end{tabular}

The results of the numerical investigation to the applied atmosphere models are shown in Fig. 12. The pressure distributions point out that a maximum uncertainty of approximately $14 \%$ in

\footnotetext{
${ }^{1}$ Global Reference Atmospheric Model - 1999

${ }^{2}$ COSPAR (Committee on Space Research) International Reference Atmosphere -1986
}

pressure may be expected which is relatively large. The results to the heat flux and temperature pointed out that the differences in the selected atmosphere models have a smaller consequence, because their maximum uncertainties are approx. $3 \%$ (heat flux) and $1 \%$ (temperature) respectively. For the following calculations the US Standard Atmosphere 1976 is applied.
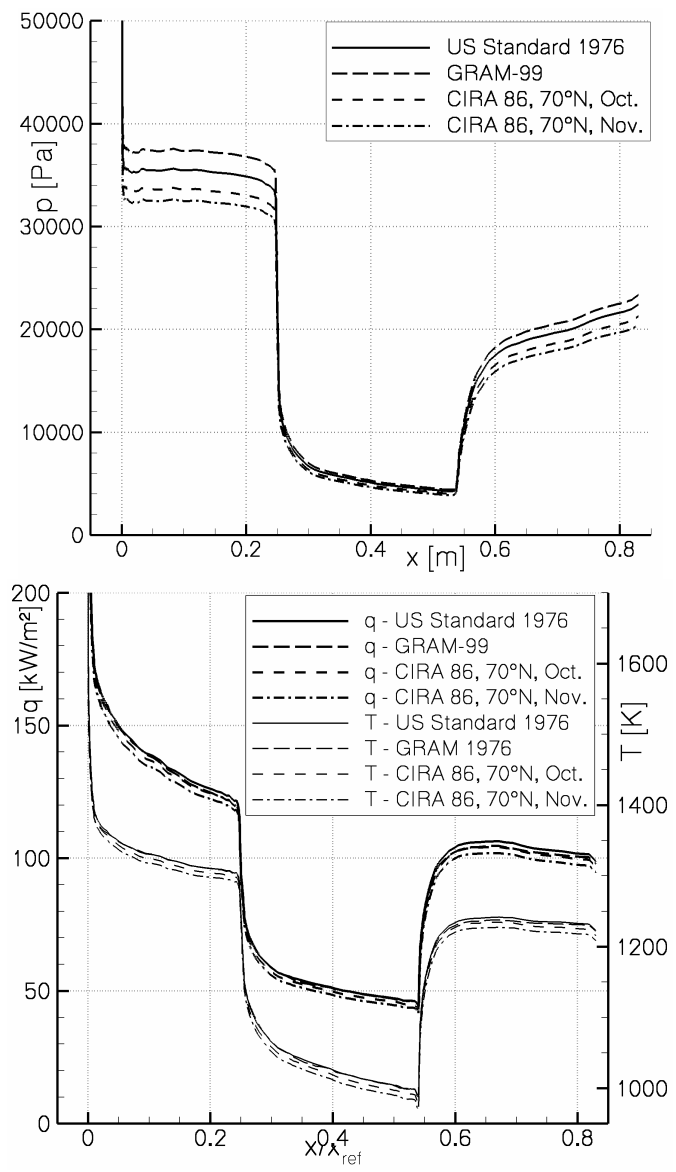

Fig. 12 Comparison of different atmosphere models (Alt $=20 \mathrm{~km}, \mathrm{M}=6.2, \alpha=0^{\circ}$ ) on pressure (top) and temperature and heat fluxes (bottom)

\subsection{Numerical investigations of the re-entry} In this subsection a comparison of numerical and experimental data (surface pressure and heat flux) for selected altitudes along the re-entry flight on only the first segment of SHEFEX I (see Fig. 7) are carried out. On this part of the configuration only one pressure port and one heat flux sensor are available.

Since all previous results have been achieved assuming radiation adiabatic walls, at first the possible kind of modelling of the thermal wall behaviour for the simulation is analysed in detail. For this purpose Fig. 13 shows the materials of 
SHEFEX-TPS and that of the included heat flux sensor. The TPS consists of the DLR's C/C-Sic material, which has demonstrated very moderate erosion behaviour at extreme heat loads in the past [Eggers (2006)]. On the other hand the heat flux sensor consists of a combination of Nickel housing and Nichrome/Constantan thermopile. Because of that the thermal properties of the TPS and the sensor are different. Therefore, both materials will be heated-up at different speed and their wall temperatures have to be considered separately for the numerical simulations.

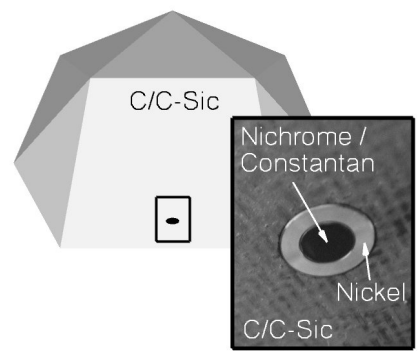

Fig. 13 Material composition of SHEFEX I

For the calculations a constant temperature at wall it is assumed and the computations are done for a fixed time without coupling with the structure. The required temperatures of the TPS and the sensor for the numerical simulations were measured during the re-entry. Fig. 14 shows the calculation for Alt $=22 \mathrm{~km}$ as an example. The heat flux at the leading edge is extremely high and decreases along the lower surface. At the position of the sensor the heat flux escalates due to the smaller wall temperature of the sensor $\left(\mathrm{T}_{\text {Sensor }}=\right.$ $390 \mathrm{~K})$ compared to the temperature of the TPS $\left(\mathrm{T}_{\mathrm{C} / \mathrm{C}-\mathrm{Sic}}=500 \mathrm{~K}\right)$.

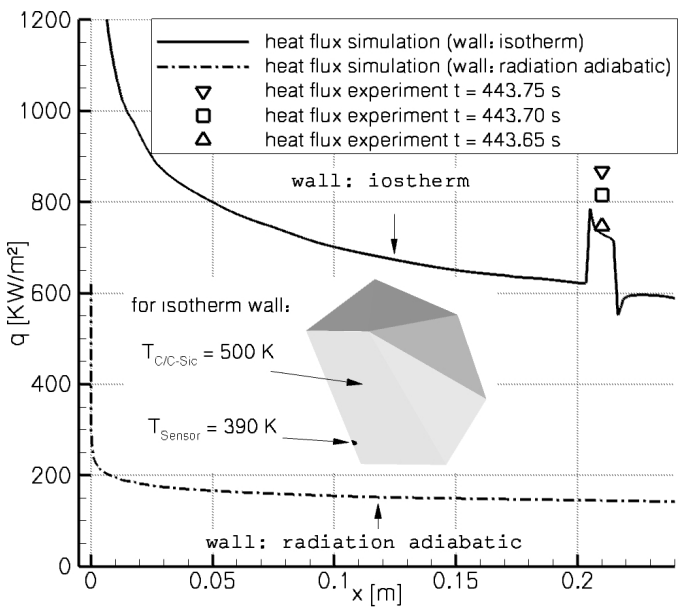

Fig. 14 Influence of wall boundary condition on heat flux distribution $\left(\mathrm{Alt}=22 \mathrm{~km}, \mathrm{M}=6.2, \alpha=2.4^{\circ}\right.$ )
Furthermore, Fig. 14 shows that is not possible to define the thermal behaviour of SHEFEX I as radiation adiabatic. In that case the heat flux distribution appears definitely too small. Therefore, in the following simulations the boundary condition of the wall is defined as isotherm using the measured wall temperatures of the flight.

The investigation of the re-entry includes an altitude range from 50 down to $20 \mathrm{~km}$, which SHEFEX I has flown through in approximately 17 seconds. Here, the Mach number varies between 5.4 and 6.2. The angle of attack oscillates between -5.0 and 4.1 degree. In order to reduce the calculation effort only altitudes with sideslip angle smaller than $3^{\circ}$ are selected within the range $20-50 \mathrm{~km}$ so that half of the SHEFEX I first segment may be computed. Table 3 contains all the parameters required to simulate the flow at the chose altitudes.

Table 3 Selected altitudes of re-entry for simulations

\begin{tabular}{|c|c|c|c|c|}
\hline $\begin{array}{c}\mathbf{t} \\
{[\mathbf{s}]}\end{array}$ & $\begin{array}{c}\text { Alt } \\
{[\mathbf{k m}]}\end{array}$ & $\begin{array}{c}\mathbf{M} \\
{[-]}\end{array}$ & $\begin{array}{c}\boldsymbol{\alpha}^{\prime} \\
\left.{ }^{\circ}\right]\end{array}$ & Boundary layer \\
\hline 427.75 & 50.0 & 5.4 & -4.5 & laminar \\
\hline 430.45 & 45.4 & 5.5 & -4.0 & laminar \\
\hline 432.35 & 42.0 & 5.7 & -2.9 & laminar \\
\hline 433.80 & 39.5 & 5.8 & -5.0 & laminar \\
\hline 436.05 & 35.4 & 6.0 & -3.4 & laminar \& turbulent \\
\hline 438.50 & 31.0 & 6.1 & 3.7 & laminar \& turbulent \\
\hline 440.00 & 28.3 & 6.2 & 4.1 & laminar \& turbulent \\
\hline 441.50 & 25.6 & 6.2 & -0.6 & laminar \& turbulent \\
\hline 442.35 & 24.1 & 6.2 & 1.2 & turbulent \\
\hline 443.35 & 22.3 & 6.2 & 0.1 & turbulent \\
\hline 443.95 & 21.3 & 6.2 & 2.5 & turbulent \\
\hline 444.50 & 20.3 & 6.1 & 1.2 & turbulent \\
\hline
\end{tabular}

Fig. 15 shows a comparison of experimental and numerical data for the pressure and heat flux distributions. In general it can be noted that for altitudes above $28 \mathrm{~km}(440 \mathrm{~s})$ the atmospheric effects were quite small wherefore the pressure and heat flux values were relatively minor. The changing angle of attack caused a corresponding oscillated pressure and heat flux values. Particularly below $28 \mathrm{~km}$ the angle of attack has an increasing influence on the analysed data. 

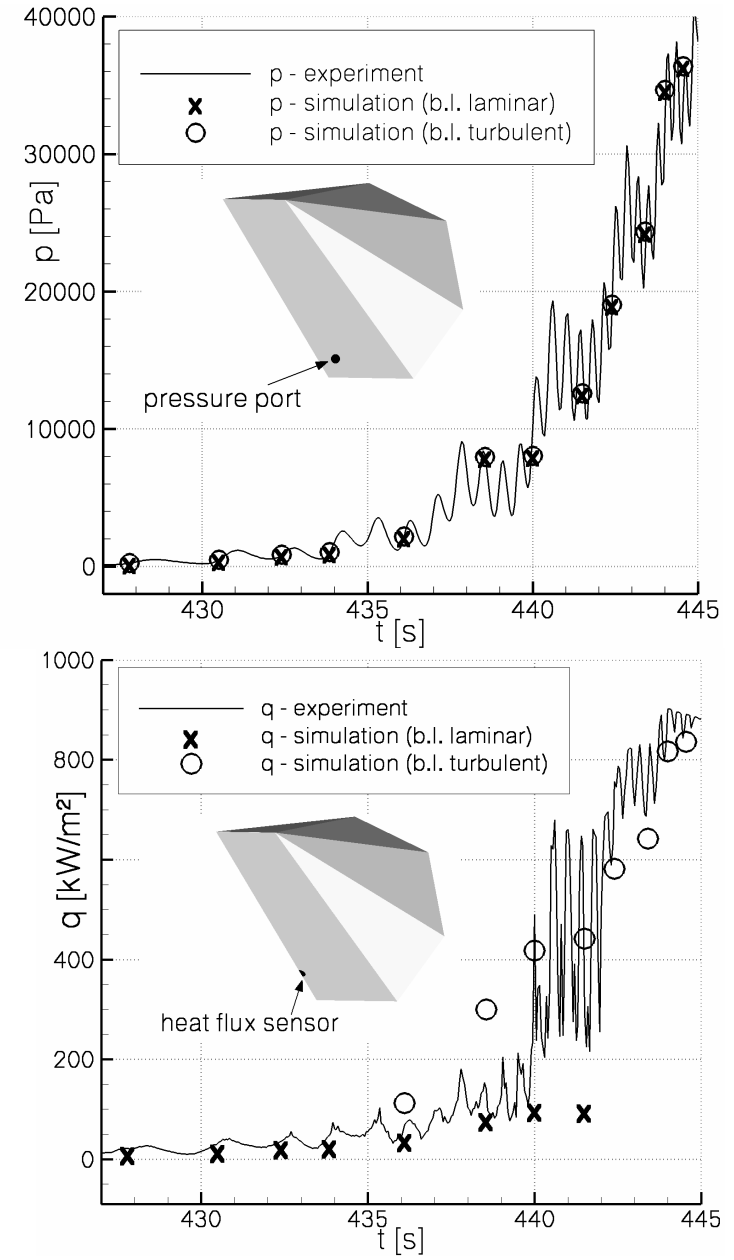

Fig. 15 Comparison of numerical and experimental data (surface pressure $p$ and heat flux $q$ ) for altitudes according Table 3

The surface pressure is well captured by the numerical results for the whole investigated altitude range and is nearly independent of the applied boundary layer (laminar / turbulent). The pressure increases from 3 to $40 \mathrm{kPa}$. The distribution of the heat flux is qualitatively equal to the pressure distribution. Initially, the values are quite small (below $100 \mathrm{~kW} / \mathrm{m}^{2}$ ) and increase after this to $900 \mathrm{~kW} / \mathrm{m}^{2}$. As explained in chapter 3 , the TAU code does not model transition from laminar to turbulent flow. However, by comparing the computed heat fluxes for laminar and turbulent boundary layer with the measured values it is possible to infer that transition took place approximately at $28 \mathrm{~km}$. At this altitude and below the experimental values compare better with the turbulent simulations.

Although the calculation delivers lower heat fluxes than measured in flight, the agreement is inside the uncertainties on the well modelling parameters.

\section{CONCLUSIONS}

In this study an aero- and thermodynamic analysis for SHEFEX I has been performed. A couple of carefully investigations to the accuracy of the data recording and to the potentials source of error for the numerical simulations like influence of sideslip, differences between $2 \mathrm{D}$ and $3 \mathrm{D}$ simulations, impact of ablation, influence of atmosphere model, and so on have been carried out. It can be summarized that the experimental data and flight data recording during the experiment delivers synchronous values, which is very important for the post-flight analysis using numerical simulations. The sensitivity analysis shows that there are a lot of parameters and modelling conditions which have an impact on the numerical analysis of SHEFEX I. In particular a major observation is that the radiation adiabatic wall condition is inappropriate for the analysis of the present experiment.

Considering all investigated sources of errors the numerical results for the pressure and heat flux distributions at selected altitudes of the re-entry offer a well agreement with the experimental data.

\section{ACKNOWLEDGEMENTS}

The author would like to thank indeed J.M.A. Longo for his help and support in context with this work.

\section{REFERENCE}

1. Barth, Tarik; Eggers, Thino: SHEFEX - A First Aerodynamic Post-Flight Analysis, 15. DGLR-Fach-Symposium AG STAB, Darmstadt (de), 2007

2. Dankert, Carl; Otto, Hannes: Experimental Investigation and Numerical Simulation on a Missile Radome at Mach 6, 15. DGLR-FachSymposium AG STAB, Darmstadt (de), 2006

3. Gardner, A.D.; Hannemann, K.; Steelant, J.; Paull, A., Ground Testing of the HyShot Supersonic Combustion Flight Experiment in HEG and Comparison with Flight Data, AIAA-Paper 2004-3345, 2004.

4. Eggers, Th.; Longo, J.M.A.; Turner, J.; Jung, W.; Hörschgen, M.; Stamminger, A.; Gülhan, A.; Siebe, F.; Requardt, G.; Laux, T.; Reimer, T.; Weihs, H.: The SHEFEX Flight Experiment - Pathfinder Experiment for a Sky Based Test Facility, AIAA 2006-7921, 2006. 
5. Gülhan, A.; Requardt, G.; Siebe, F.; Koch, U.; Esser, B.: Instrumentation and In-Flight Data of the SHEFEX Flight Experiment, Proceedings of the $1^{\text {st }}$ International ARA Days on Atmospheric Re-Entry Systems, Missions and Vehicles, Arcachon, France, June 2006.

6. Johnston, I.A.; Weiland, M.; MartinezSchramm, J.; Hannemann, K.; Longo, J.M.A.: Aerothermodynamics of the ARD: Post-Flight Numerics and Shock-Tunnel Experiments, AIAA Paper 2002-0407, 2002.

7. Schramm Martinez, Jan; Reimann, Bodo: Aerothermodynamic Investigation of the Pre$X$ Configuration in HEG, 15. DGLR-FachSymposium AG STAB, Darmstadt (de), 2007 